\section{Composition of Vibrations}

WHILE holding one of König's large polished tuning-forks in my hand, $I$ happened to give it a swaying movement on the plane on which its vibrations were being performed, and immediately noticed that the space through which the fork swing was occupied by a series of bright straight lines arranged in a fan-like form. The lines spread out, or drew together, as the rate of movement impressed on the fork increased or diminished. The case was clearly one of composition of vibrations, the bright lines being merely the edges of the prongs seen in positions of instantaneous rest, where the proper motion of a prong was equal and opposite to that communicated to it by the hand.

By taking forks of different pitch, and causing them to swing with equal velocities, the dependence of pitch on the number of vibrations performed in a given time was easily exhibited.

In case this simple observation has not yet been made or desclibed I ask its insertion in NATURE.

Trinity College, Cambridge

\section{SeDley TayloR}

\section{Eclipse Photography}

Mr. J. Boesinger, in the last number of NATURE, expresses his surprise at the ignorance of the photographers attached to the late expeditions, and favours them with hints, observations, and instructions still more surprising. Because he cannot see their reasons for employing equatorial stands, plates in separate frames, and long exposures, he concludes these were unnecessary; and affirms "there must have been a great want of balance in their chemicals." No doubt there is a want of balance somewhere, and I diffidently sulmit the probability that Mr. Boesinger has lost his.

I would briefly state to those few of your readers who may have been misled by this correspondent, that equatorial stands driven by clock-work are absolutely necessary in the production of the best results, either by short or long exposure of photographic plates; a picture "not perfectly sharp but valuable as a memorial," was what Mr. Boesinger aimed at (and I sincerely hope he obtained it), but the expeditions had higher aims and greater expectations. Single large plates were exposed separately, that should a corona extending many degrees be actinically present, it might find ample room to put in an appearance; in such a case had "repeating backs" been used to give many pictures on one plate, there would have been great danger from the corona of one picture over- lapping that of another, to the ruin of all. Comparatively long exposures were found necessary to secure impression from the faint extremities of the rays.

HENRY DAVIS

Tidal Friction according to Thomson and Tait

I AM so afraid that this letter will convict me of hopeless stupidity that I conceal my name. For I am going to confess that I do not understand, and even feel inclined to dispute, the reasoning of Thomson and Tait, on pp. 191-194 in their great work, respecting the effect of tidal friction on the motion of the e irth and moon. It will be a convenience to your readers if $I$ quote the passage at full length :-

"Let us suppose the moon to be a uniform spherical body. The mutual action and reaction of gravitation between her mass and the earth's will be equivalent to a single force in some line through her centre, and must be such as to impede the earth's rotation as long as this is performed in a shorter period than the rotation as long as this is performed motion round the earth. It must therefore lie in some such direc-

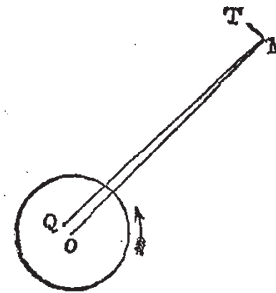

tion as the line MQ in the diagram, which represents, necessarily with enormous exaggeration, its deviation, $\mathrm{OQ}$, from the earth's centre. Now, the actual force on the moon in the line $\mathrm{MQ}$, may be regarded as consisting of a force in the line MO towards the earth's centre, sensibly equal in amount to the whole force, and a comparatively very small force in the line MT perpendicular to MO. This latter is very nearly tangential to the moon's path, and is in the direction with her motion. Such a force, if suddenly commencing to act, would, in the first place, increase the moon's velocity; but after a certain time she would have moved so much farther from the earth, in virtue of this acceleration, as to have lost, by moving against the earth's attraction as much velocity as she had gained by the tangential accelerating force."

The consequences are then shown to be that the moon's distance would be increased in the ratio $I: I .46$, and her periodic time increased, and the earth's period of rotation lengthened.

This reasoning perplexes me; for if the effect of a certain amount of fluid friction is to throw the line of action of the force from $\mathrm{MO}$ to $\mathrm{MQ}$, a fuid friction is conceivable which should throw it outside the earth altozether. Moreover, the line of attraction of the earth on the moon would be in a line not passing through the earth's centre, a result I cannot understand, especially if the fluid friction were increased as just suggested. Nor can $I$ see that a force in $M Q$, the centre of the earth keing free, would tend to stop the rotation of the earth.

As I view the matter, fluid friction generates a couple tending to stop the rotation of the earth, and it is impossible to combine this couple with the force in MO, and represent the resultant by a single force. The energy lost in the form of momentum of rotation of the earth is gained in the heat devolved by the fluid friction, which is ultimately dissipater. And the final result would be that the orbit of the moon would not be appreciably altered, while the period of rotation of the earth is gradually lengthened.

Am I wrong, for the thousandth time in my life? and if so will some one try and enlighten me. Pelhaps Prof. Tait will spare a few minutes to an old friend.

M. A.

\section{Circumpolar Lands}

In Nature (Feb. 8) Mr. Murphy seems to admit the soundness of the reasoning by which I endeavoured to show (Jan. 25) that the earth's form is probably undergoing a slow progressive change, but he thinks that the statements in the first and last parts of my letter are contradictory.

If Mr. Murphy will be good enough to read again the paragraph immediately following the one which he quotes, I think he will find that there is no contradiction. "Transmission of pressure towards the poles " must tend to elevate the land in those regions. How that pressure is produced and transmitted I have endeavoured to show in the same paragraph.

However, the main proposition which I sought to establish in my paper of 1857 , before alluded to, is that any spheroid of equilibrium, whether earth, sun, or any other, in motion about an axis, in cooling from a flud state, undergoes a change of form, and with this proposition Mr. Murphy seems to agree.

Mr. Murphy has inadvertently omitted part of a sentence in making his quotation from my letter, thus representing me as spenking of a ratio with one quantity only.

Queen's Coll., Liverpuol, Feb. I6 George Hamilton

\section{The Spheroidal State of Water}

I HAD the pleasure a few days ago of visiting Messrs. Johnson's celebrated iron wire manufaclory in Manchester. There may be seen a series of furnaces and rolling mills which in twenty-four hours can convert a truck load of the best Swedish iron into the bright and polished galvanised wire which is now being so extensively employed to complete our very perfect system of Post Office telegraphs. Every stage of the process passes beneath the eye of the observer; the melting of the pigs, the formation of the billets, the puddling of the bloom, the shingling of the balls, the rolling of the bars, and their subsequent extension by further rolling, and drawing into telegraph wire.

The bars are cut off into loft. lengths, and are placed in a Siemen's regenerative furnace, where they are raised to a brilliant white heat. They are then drawn out of the mouth of the glow. ing furnace, and pass through a series of consecutive rollers of varying dimensions, and rotating with varying speed, uitimately flowing out in a continuous stream of iron wire. In fact, the metal is at such a high temperature and so plastic that the curves it takes in falling convey the idea of a thin, fine unbroken jet of liquid matter.

The rollers are kept cool by the constant play upon them of jets of water. The first pair of rollers is fixed close to the mouth of the furnace, which is partially closed by a moveable screw that is only raised when the attendant sprite requires to direct another bar to the attenuating process of the continuous rollers. The jet of water that cools the first pair of rollers in one furnace fell in a broken shower upon the foot-plate of the mouth of the furnace, which, from its proximity to the fire, was raised to a 